\title{
Pharmacodynamic Assessment of Amoxicillin-Sulbactam Against Acinetobacter baumannii: Searching the Optimal Dose and Infusion Time Through a Human ex-vivo Model
}

\author{
Carlos Bantar ${ }^{1}$, Liliana Fernández Canigia ${ }^{2}$, María Alejandra Berger ${ }^{2}$, Jorge L Soutric ${ }^{3}$ and Héctor $\mathbf{J}^{\text {Arenoso }}{ }^{3}$ \\ ${ }^{1}$ Laboratorio Domingo I Nanni; Entre Rios, PR, Argentina; ${ }^{2}$ Laboratorio de Microbiología, Hospital Alemán and ${ }^{3}$ Laboratorios Bagó S.A.; \\ Buenos Aires, Argentina
}

\begin{abstract}
Amoxicillin-sulbactam (AMX-SUL) is an aminopenicillin/ß-lactamase inhibitor combination currently available in 29 countries and may be a suitable option for treating infections caused by Acinetobacter spp. Thus, we sought to search the optimal dosing strategy for this formulation through an ex vivo pharmacodynamic human model against Acinetobacter baumanniii. Four volunteers were randomized to receive alternatively a single dose AMX-SUL infused both either over $30 \mathrm{~min}$ or $3 \mathrm{~h}$ at the following ratios $(\mathrm{g} / \mathrm{g}): 1 / 0.5 ; 1 / 1$, and $0 / 2$. Time-kill studies were performed with

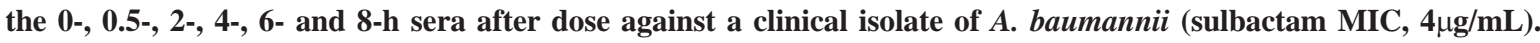
Bactericidal activity (i.e. a mean decrease $\geq 3 \log _{10} \mathrm{CFU} / \mathrm{mL}$ in the viable cell counts from the initial inoculum) was displayed by the 0.5 - and the 2-h sera after dose for all formulations. The 4-h sera proved inhibitory with the AMXSUL $1 \mathrm{~g} / \mathrm{g}$ formulation, albeit a trend to regrowth was observed after 24-h incubation. With the AMX-SUL 0g/2g dose, the 4-h sera proved almost bactericidal activity (i.e. a mean decrease of $2.4 \log _{10} \mathrm{CFU} / \mathrm{mL}$ in the viable cell counts from the initial inoculum), whereas the 6-h sera was inhibitory, with a trend to regrowth after 24-h incubation. When infused over $3 \mathrm{~h}, \mathrm{AMX}-\mathrm{SUL} 1 \mathrm{~g} / 0.5 \mathrm{~g}$ and $1 \mathrm{~g} / \mathrm{g}$, bactericidal activity was displayed by the 0.5-, 2- and the 4-h sera after dose and the 6-h sera proved inhibitory with the AMX-SUL 1g/1g formulation. The present study, albeit preliminary, might give a rationale for the dosing strategy to treat infections caused by $A$. baumannii with sulbactam, either alone or combined with amoxicillin. A 2-g sulbactam dose seems to be optimal to be infused over 30 min with a 6-h dosing interval. When infused over $3 \mathrm{~h}$, AMX-SUL $1 \mathrm{~g} / 1 \mathrm{~g}$ given every $6 \mathrm{~h}$ or $8 \mathrm{~h}$ seems a suitable dosing schedule. Key-Words: Amoxicillin-sulbactam, Acinetobacter.
\end{abstract}

Acinetobacter baumannii is becoming an important pathogen associated with nosomial infections worldwide [1]. It is invariably multiresistant and one major concern is the increasing incidence of carbapenem-resistant isolates, since these antibiotics are frequently the therapeutic choice for these infections [2]. Carbapenem resistance in South America has already reached levels that significantly influence therapeutic choices $[3,4]$ and this looks set to be a problem in several other regions $[5,6]$. Acinetobacter spp. has been found at the fourth rank in hospitalized patients with pneumonia in Latin America [6] and at the second one in patients with nosocomial pneumonia in Argentina [7], with carbapenem resistance increasing dramatically [4], especially in Argentina [4,7] and in Colombia [4,8]. This kind of resistance has also been reported at modest rates in Chile [4] and Brazil [4,9], albeit it seems to remain stable in some regions of this late country [10]. As a result, old antibiotics, such as colistin and ampicillinsulbactam, have emerged as the obligate alternative for treating serious infections associated with A. baumannii [11].

Both early [12] and present [13] evidences suggest that sulbactam is active in vitro against Acinetobacter spp, whereas some reports showed that this drug, given as a combination of ampicillin-sulbactam, may yield acceptable clinical success when treating infections associated with this

Received on 13 April 2009; revised 28 September 2009.

Address for correspondence: Dr.Carlos Bantar. Colón 128, (3100) Paraná, Entre Ríos.Fax: (54343)4310783. E-mail: cbantar@arnet.com.ar. This study was supported by a grant from Laboratorios Bagó S.A., Buenos Aires, Argentina.

The Brazilian Journal of Infectious Diseases

2009;13(5):348-352. (C) 2009 by The Brazilian Journal of Infectious Diseases and Contexto Publishing. All rights reserved. pathogen $[11,14]$. However, neither large controlled clinical trials, nor pharmacodynamic studies have been undertaken to assess the efficacy of sulbactam against Acinetobacter spp $[11,14]$. Amoxicillin-sulbactam (AMX-SUL) is an aminopenicillin/ $\beta$-lactamase inhibitor combination marketed in Argentina since 1988 and is currently available in 29 countries. Indeed, more than two millions of patients with either urinary or respiratory tract infections have been treated by oral route with this formulation from 1988 to 2007 only in Argentina (data from files of Laboratorios Bagó S.A., Argentina). Although we have described the pharmacodynamics of AMX-SUL oral tablets thoroughly [1518], the intravenous formulation of this drug have not been assessed, as the intravenous combination was allowed to be marketed on the basis of the pharmacokinetics and pharmacodynamics ( $\mathrm{pk} / \mathrm{pd}$ ) tested for the oral formulation. As a result, neither pk/pd studies, nor clinical trials were required for the approval of intravenous AMX-SUL. Because of intravenous AMX-SUL may be a suitable option for treating infections caused by Acinetobacter spp, we sought to search the optimal dose of sulbactam to be used in this formulation through an ex vivo pharmacodynamic human model. In addition, the benefit of an extended infusion was assessed.

\section{Material and Methods}

Study Design

This was an experimental, randomized, single-blind, 4-way crossover intra-subject study. It was conducted following the principles of the Declaration of Helsinki on Good Clinical Practice and received approval of an independent Ethics Committee and the national health authorities (A.N.M.A.T, 
authorization $\mathrm{N}^{\mathrm{o}}$ 3203/06). Informed consent was obtained from the volunteers before enrollment.

\section{Subjects, Antimicrobial Dose and Sampling}

After an overnight fasting, 4 healthy subjects, 1 male and 3 female; mean age, 35.0 years (range 27 to 51 years) and mean weight, $60.5 \mathrm{~kg}$ (range 46.0 to $75.0 \mathrm{~kg}$ ), were randomized to receive alternatively (i.e. separated by a 7-days washout period) the different doses. AMX-SUL (Trifamox IBL Inyectable Laboratorios Bagó SA, Buenos Aires, Argentina) was given diluted in $100 \mathrm{~mL}$ of sterile saline solution as a single dose infused over $30 \mathrm{~min}$ at the following amoxicillin/ sulbactam ratios (g/g): 1/0.5; $1 / 1$ and $0 / 2$. In addition, after a 15-days period, AMX-SUL, both at $1 \mathrm{~g} / 0.5 \mathrm{~g}$ and at $1 \mathrm{~g} / 1 \mathrm{~g}$ formulation, was infused over 3 h to the 4 volunteers separated by a 7-days washout period. Venous blood samples were obtained immediately before (time zero, control) and at 2, 4, 6 and $8 \mathrm{~h}$ after dose. Serum harvested from blood was immediately fractionated and stored at $-70^{\circ} \mathrm{C}$ until use.

\section{Antibiotic Concentration in Serum}

Levels of antibiotics were determined in all serum samples by a bioassay method as previously described [13]. The Bacillus subtilis ATCC 6633 strain and a clinical isolated of $A$. baumannii (strain 5355-81, sulbactam MIC, $2 \mu \mathrm{g} / \mathrm{mL}$ ) were used for testing amoxicillin and sulbactam, respectively. The lower limit of detection was $0.12 \mu \mathrm{g} / \mathrm{mL}$ for amoxicillin and $2.5 \mu \mathrm{g} / \mathrm{mL}$ for sulbactam. Percent coefficient of variation ranged between $9 \%$ and $15 \%$. The linearity of every standard curve was assured by a regression coefficient (r) of at least 0.998 .

\section{Time-Kill Studies}

Time-kill studies were performed as described previously [15] with the 0- (growth control), 2-, 4-, 6- and 8-h sera after dose against a clinical isolate of $A$. baumannii (sulbactam MIC, $4 \mu \mathrm{g} / \mathrm{mL}$ ). Briefly, serum samples were inoculated with equal volumes of Mueller-Hinton broth (Difco, Detroit, Mich.) containing the organism at a concentration of roughly $1 \times 10^{6}$ $\mathrm{cfu} / \mathrm{mL}$ in log phase. All tubes were incubated at $35^{\circ} \mathrm{C}$ in room atmosphere. Viable cell counts were performed after 0, 2, 4, 6, 8 and 24 h of incubation to establish a 24-h time-kill curve. Bactericidal activity was defined as a $\geq 3-\log _{10} \mathrm{cfu} / \mathrm{mL}$ decrease in the viable cell counts regarding the original inoculum. Inhibitory activity was defined as $<3-\log _{10} \mathrm{cfu} / \mathrm{mL}$ decrease in viable cell counts. Any viable cell count higher than the starting inoculum was considered as overgrowth. Carryover of antibiotics was avoided by 10 -fold serial dilutions before plating. The lower limit of detection was $100 \mathrm{cfu} / \mathrm{mL}$ and this value was assigned to any count dropping below this limit.

\section{Data Analysis and Statistics}

Levels of antibiotic in serum are given as mean values and standard deviation (SD) calculated from the 4 volunteers. Likewise, for sera corresponding to the different times after dose from all volunteers, an average time-kill curve was constructed for every AMX-SUL dose with the mean values of viable cell counts $\left(\log _{10} \mathrm{cfu} / \mathrm{mL}\right)$ obtained at the different times of incubation (i.e. 0, 2, 4, 6, 8 and 24h).

\section{Results}

Table 1 shows the mean serum levels of amoxicillin and sulbactam after the infusion of AMX-SUL at different ratios between components, infused both over $30 \mathrm{~min}$ and $3 \mathrm{~h}$. When infused over $30 \mathrm{~min}$, the formulation AMX-SUL 1g/1g yielded higher amoxicillin and sulbactam levels than AMX-SUL 1g/ $0.5 \mathrm{~g}$ at every time after dose, whereas AMX-SUL $0 \mathrm{~g} / 2 \mathrm{~g}$ reached the highest sulbactam concentrations. When infused over 3h, AMX-SUL (both 1g/0.5g and $1 \mathrm{~g} / 1 \mathrm{~g}$ ) yielded amoxicillin and sulbactam levels at $2 \mathrm{~h}$ and $4 \mathrm{~h}$ after dose higher than the respective values observed with any AMX-SUL formulation infused over $30 \mathrm{~min}$, with the formulation $1 \mathrm{~g} / 1 \mathrm{~g}$ showing the highest levels. Furthermore, sulbactam concentration obtained at $2 \mathrm{~h}$ and $6 \mathrm{~h}$ after dose with AMX-SUL $1 \mathrm{~g} / 1 \mathrm{~g}$ infused over $3 \mathrm{~h}$ was higher than the respective values observed with $2 \mathrm{~g}$ of sulbactam alone infused over $30 \mathrm{~min}$.

Average time-kill curves of serum from the 4 volunteers receiving the different AMX-SUL formulations over $30 \mathrm{~min}$ against $A$. baumannii 5355-81 are given in Figure 1 . Bactericidal activity (i.e. a mean decrease $\geq 3 \log _{10} \mathrm{CFU} / \mathrm{mL}$ in the viable cell counts from the initial inoculum) was displayed by the 0.5- and the 2-h sera after dose for all formulations. The 4-h sera proved inhibitory with the AMX-SUL $1 \mathrm{~g} / 1 \mathrm{~g}$ formulation (Figure 1B), albeit a trend to regrowth was observed after 24$\mathrm{h}$ incubation. With the AMX-SUL 0g/2g dose, the 4-h sera proved almost bactericidal activity (i.e. a mean decrease of 2.4 $\log _{10} \mathrm{CFU} / \mathrm{mL}$ in the viable cell counts from the initial inoculum), whereas the 6-h sera was inhibitory, with a trend to regrowth after 24-h incubation (Figure 1C). The remaining serum samples collected after dose were unable to exert significant antimicrobial effect with any formulation.

Average time-kill curves of serum from the 4 volunteers receiving the AMX-SUL $1 \mathrm{~g} / 0.5 \mathrm{~g}$ and $1 \mathrm{~g} / 1 \mathrm{~g}$ combinations infused over 3h against are given in Figure 2. Bactericidal activity was displayed by the 0.5-, 2- and the 4-h sera after dose. The 6-h sera proved inhibitory with the AMX-SUL 1g/ $1 \mathrm{~g}$ formulation (fig. 2B), albeit a trend to regrowth was observed after 24-h incubation.

\section{Discussion}

Several efforts are being made to assess alternative options for treating infections associated with multidrug-resistant $A$. baumannii and both early and newer antibiotics have been tested [11]. Ampicillin-sulbactam is one of these early drugs proving in vitro activity [12] and two recent comparative, one retrospective [14] and one prospective [19], studies suggest that it may be effective against infections caused by this organism. In the retrospective study, more than 80 patients were either treated with colistin or ampicillin-sulbactam and colistin, but not ampicillin-sulbactam, proved independently associated with higher mortality rate [14]. In the prospective 
Table 1. Serum levels of different amoxicillin-sulbactam (AMX-SUL) formulations infused to 4 volunteers by intravenous route over 30 min and $3 \mathrm{~h}$.

\begin{tabular}{lccccc}
\hline $\begin{array}{l}\text { Component and } \\
\text { time of infusion }\end{array}$ & \multicolumn{4}{c}{ Mean \pm SD concentration $(\mu \mathbf{g} / \mathbf{m L})$ at the following time after dose } \\
\cline { 2 - 6 } Over 30 min & $\mathbf{0 . 5 h}$ & $\mathbf{2 h}$ & $\mathbf{4 h}$ & $\mathbf{6 h}$ & $\mathbf{8 h}$ \\
AMX-SUL 1g/0.5g & & & & & \\
$\quad$ & & & & \\
$\quad$ AMX & $42.7 \pm 28.9$ & $4.6 \pm 3.0$ & $0.76 \pm 0.44$ & $0.25 \pm 0.13$ & $<0.12$ \\
$\quad$ SUL & $25.8 \pm 11.3$ & $3.8 \pm 1.50$ & $<2.5$ & $<2.5$ & $<2.5$ \\
AMX-SUL 1g/1g & & & & & \\
$\quad$ AMX & $57.5 \pm 41.6$ & $6.1 \pm 4.1$ & $1.0 \pm 0.94$ & $0.36 \pm 0.94$ & $<0.12$ \\
$\quad$ SUL & $50.8 \pm 22.9$ & $8.63 \pm 3.81$ & $2.54 \pm 0.14$ & $<2.5$ & $<2.5$ \\
AMX-SUL 0g/2g & & & & & \\
$\quad$ SUL & $98.1 \pm 5.78$ & $24.1 \pm 11.4$ & $5.5 \pm 3.3$ & $<2.5$ & $<2.5$ \\
Over 3h & & & & & \\
AMX-SUL 1g/0.5g & & & & & \\
$\quad$ AMX & $43.4 \pm 28.8$ & $52.5 \pm 31.7$ & $3.1 \pm 2.1$ & $0.50 \pm 0.42$ & $0.18 \pm 0.10$ \\
$\quad$ SUL & $29.0 \pm 18.6$ & $41.0 \pm 23.3$ & $4.0 \pm 1.71$ & $<2.5$ & $<2.5$ \\
AMX-SUL 1g/1g & & & & & \\
$\quad$ AMX & $50.4 \pm 53.5$ & $75.8 \pm 54.2$ & $5.2 \pm 1.3$ & $0.60 \pm 0.50$ & $0.3 \pm 0.23$ \\
$\quad$ SUL & $30.4 \pm 29.4$ & $59.0 \pm 33.5$ & $5.1 \pm 0.14$ & $3.1 \pm 0.25$ & $<2.5$ \\
\hline
\end{tabular}

Figure 1. Average time-kill curve of serum from 4 volunteers receiving intravenous AMX-SUL; $1 \mathrm{~g} / 0.5 \mathrm{~g}(\mathrm{~A}), 1 \mathrm{~g} / \mathrm{g}(\mathrm{B})$ and $0 \mathrm{~g} / 2 \mathrm{~g}(\mathrm{C})$ infused over $30 \mathrm{~min}$, against a clinical isolate of Acinetobacter baumannii (sulbactam MIC, $4 \mu \mathrm{g} / \mathrm{mL}$ ). Data are means \pm standard deviation (error bars) at the following times after dose (h): 0 ( • control), $0.50(\bullet), 2(*), 4(\bullet), 6(\mathbf{x})$ and $8(\bullet)$.

A

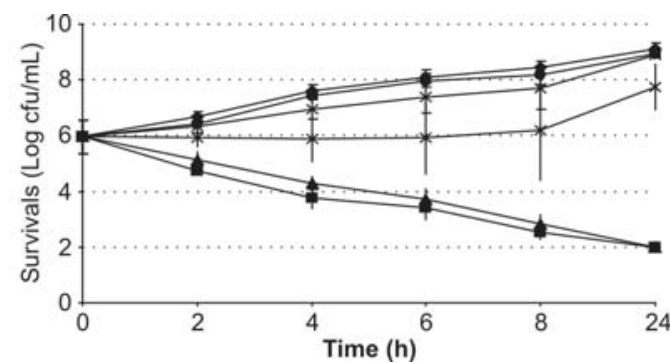

B

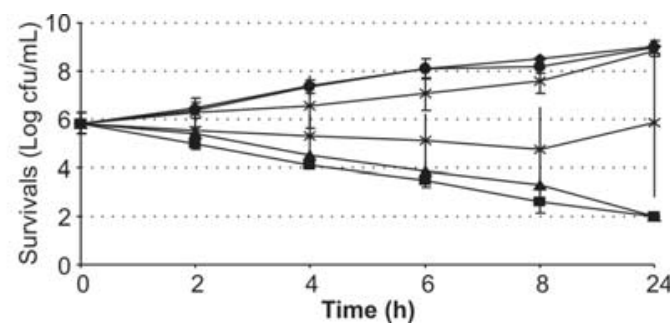

C

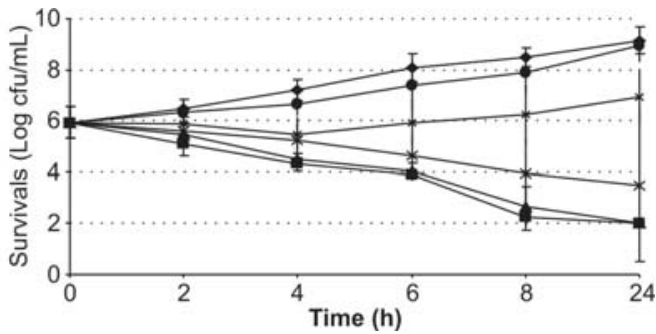

trial, 10 of 13 patients with ventilator-associated pneumonia by Acinetobacter spp displayed resolution or improvement of the symptoms and signs after treatment with high doses of ampicillin-sulbactam (i.e. $9 \mathrm{~g}$ every $8 \mathrm{~h}$ ). In addition, some case series had previously been reported [20-23]. In most studies an ampicillin-sulbactam combination containing 1-g of sulbactam, given every 8h, was used; but 2g [20] and 3g [19] of sulbactam were also employed. This dose variability may be attributable to the lack of pharmacodynamic studies for supporting any dosing schedule. We believe that determining the optimal dose is a crucial issue to reach the maximum antimicrobial efficacy with the minimal rate of certain adverse events observed in some small clinical trial testing high sulbactam dosage (i.e. 3g), such as diarrhea, skin rush or nephrotoxicity [19]. Our data show that both $0.5 \mathrm{~g}$ and $1 \mathrm{~g}$ of sulbactam, infused as AMX-SUL over $30 \mathrm{~min}$, were able to reach bactericidal serum levels at $2 \mathrm{~h}$ after dose, but a net regrowth and trend to regrowth occurred at $4 \mathrm{~h}$ after dose with the 0.5 -g and 1 -g sulbactam dose, respectively. Although these features might be enough for a 4-h dosing interval, they are insufficient to get the desirable time about MIC ( $f$ T $>$ MIC $\geq 50 \%$ ) of a 6-h schedule dosing described for a beta-lactam antibiotic [24]. By contrast, the 2-g sulbactam dose proved highly active at 4-h after dose (i.e. 67\% of a 6-h dosing interval). These findings suggest that, if $1 \mathrm{~g}$ of sulbactam is going to be used to treat an infection associated with $A$. baumannii, a dosing interval no longer than 4-h should be preferred, whereas using a 2-g dosage would allow a 6-h dosing schedule. Although we have used only one A. baumannii strain in the present study, one of us has recently demonstrated the bactericidal property of sulbactam against several clones of this organism at concentrations equal or higher than the MIC values [25]. 
Figure 2. Average time-kill curve of serum from 4 volunteers receiving intravenous AMX-SUL; 1 g/0.5g (A) and 1g/1g (B) infused over 3h, against a clinical isolate of Acinetobacter baumannii (sulbactam MIC, $4 \mu \mathrm{g} / \mathrm{mL}$ ). Data are means \pm standard deviation (error bars) at the following times after dose (h): 0 (•, control), $0.50(\mathbf{}), 2(*), 4(\boldsymbol{\Delta}), 6(\mathbf{x})$ and $8(\bullet)$.

A

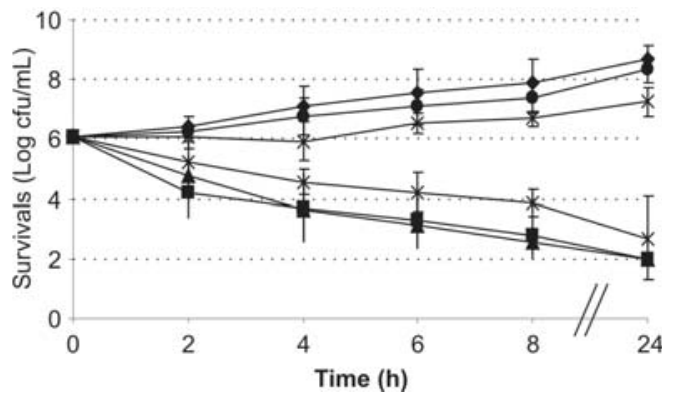

B

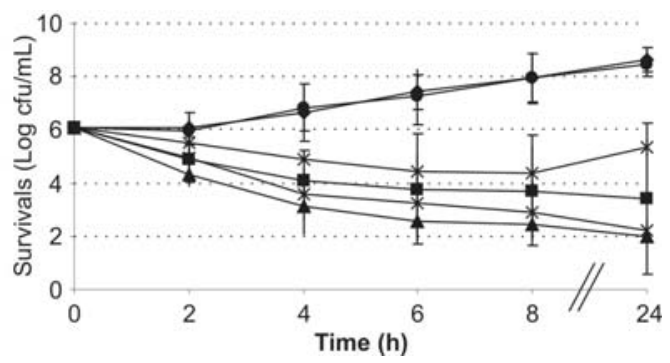

Interestingly, the 3-h extended infusion enhanced the probability of reaching the desirable pharmacodynamic target. Indeed, both $0.5 \mathrm{~g}$ and $1 \mathrm{~g}$ of sulbactam proved bactericidal at $4 \mathrm{~h}$ after dose with this infusion strategy. In addition, the 8-h after dose serum of volunteers receiving AMX-SUL 1g/1g displayed inhibitory activity. Although the benefit of the extended infusion has been described for some beta-lactams, such as piperacillin/tazobactam [24,26] and meropenem [24], it has not been reported, to our knowledge, for aminopenicillin-sulbactam combinations. It should be noted that this extended-infusion strategy may not be applicable to generic products, as stability of the drug over time is a crucial requirement to be achieve to perform extended infusion and all these experiences, including the present one, have been carried out with the original trades. In fact, significant potency decrease has been recently reported for a number of generic trades of piperacillin/tazobactam [27].

In summary, the present study, albeit preliminary, might give a rationale for the dosing strategy to treat infections caused by $A$. baumannii with sulbactam, either alone or combined with amoxicillin. A 2-g sulbactam dose seems to be optimal to be infused over 30 min with a 6-h dosing interval. When infused over 3h, AMX-SUL 1g/1g given every $6 \mathrm{~h}$ or $8 \mathrm{~h}$ seems a suitable dosing schedule. Further studies may be required to definitively confirm our findings.

\section{References}

1. Bergogne-Bérézin E. and Towner K.J. Acinetobacter spp. as nosocomial pathogens: microbiological, clinical, and epidemiological features. Clin Microbiol Rev 1996; 9:148-65.

2. Lortholary O., Fagon J-Y., Hoi A.B., Slama M.A., et al. Nosocomial acquisition of multiresistant Acinetobacter baumannii: risk factors and prognosis. Clin Infect Dis 1995; 20:790-96.

3. Brown S., Bantar C., Young H-K., Amyes S. Limitation of Acinetobacter baumannii treatment by plasmid-mediated carbapenemase ARI-2. The Lancet 1998; 350:186-87.

4. Tognim, M.C., Andrade S.S., Silbert S., et al. Resistance trends of Acinetobacter spp. in Latin America and characterization of international dissemination of multi-drug resistant strains: fiveyear report of the SENTRY Antimicrobial Surveillance Program. Int J Infect Dis 2000; 8: 284-291.

5. Afzal-Shah M., and Livermore D.M. Worldwide emergence of carbapenem-resistant Acinetobacter spp. J Antimicrob Chemother 1998; 41:576-577.

6. Gales A. C., Jones R.N., Sader H.S. Global assessment of the antimicrobial activity of polymyxin B against 54731 clinical isolates of Gram-negative bacilli: report from the SENTRY antimicrobial surveillance programme (2001-2004). Clin Microbiol Infect 2006; 12:315-321.

7. Bantar C., Famiglietti A., Radice M., et al. A 7-year national survey on bacterial resistance in bronchoalveolar lavage from patients hospitalized in Argentina. Diagn Microbiol Infect Dis 2008; 60:65-9.

8. Villegas M.V., Kattan J.N., Correa A., et al. Dissemination of Acinetobacter baumannii clones with OXA-23 Carbapenemase in Colombian hospitals. Antimicrob Agents Chemother 2007; 51:2001-4.

9. Campos Furtado G.H., Teixeira Martins S., Oliveira Machado A.M., et al. Clinical Culture Surveillance of Carbapenem-Resistant Pseudomonas aeruginosa and Acinetobacter Species in a Teaching Hospital in Sao Paulo, Brazil: A 7-Year Study. Infect Control Hosp Epidemiol 2006; 27:1270-1273

10. Zavascki A.P., Soares F.C., Superti S.V., et al. Stable carbapenem susceptibility rates among multidrug-resistant Acinetobacter spp. strains in a setting of high prevalence of carbapenem-resistant Pseudomonas aeruginosa. Int J Antimicrob Agents 2007; 30:187-189.

11. Levin A.S. and Oliveira M.S. The challenge of multidrug resistance: the treatment of Gram-negative rod infections. Shock 2008; Aug 8 [Epub ahead of print].

12. Joly-Guillou M.L., Herrman J.L., Bourdelier E., Bergongne-Bérézin E. Bactericidal in-vitro activity of b-lactams and $\beta$-lactamase inhibitors, alone or associated, against clinical strains of Acinetobacter baumannii: effect of combination with aminoglycosides. J Antimicrob Chemother 1995; 6:619-629.

13. Bantar C., Schell C., Posse G., et al. Comparative time-kill study of doxycycline, tigecycline, sulbactam, and imipenem against several clones of Acinetobacter baumannii. Diagn Microbiol Infect Dis 2008;61:309-314.

14. Oliveira M.S., Prado G.V., Costa S.F., et al. Ampicillin/sulbactam compared with polymyxins for the treatment of infections caused by carbapenem-resistant Acinetobacter spp. J Antimicrob Chemother. 2008; 61:1369-75.

15. Bantar C, Nicola F, Arenoso $\mathrm{H}$ et al. Pharmacokinetics and pharmacodynamics of amoxicillin-sulbactam, a novel aminopenicillin-b-lactamase inhibitor combination, against Escherichia coli. Antimicrob Agents Chemother 1999; 43:1503-4.

16. Bantar C, Nicola F, Fernandez Canigia L, et al. Rationale for treating community-acquired lower respiratory tract infections with amoxicillin/sulbactam combination through a pharmacodynamic analysis in the setting of aminopenicillinresistant organisms. J Chemother 2001; 13: 402-6.

17. Bantar C, Nicola F, Arenoso H, Soutric J, Caruso N, Fernández Canigia L. An ex vivo pharmacodynamic study comparing bactericidal activity of amoxicillin/sulbactam, azithromycin, doxycycline and levofloxacin against Streptococcus pneumoniae. J Chemother 2004; 16:248-54. 
18. Soutric J, Bantar C, Caruso N et al. Review of pharmacokinetic, pharmacodynamic and clinical studies with a modern combination of amoxicillin/sulbactam. Chemotherapy 2006; 52:200-4.

19. Betrosian A.P., Frantzeskaki F., Xanthaki A., Douzinas E.E. Efficacy and safety of high-dose ampicillin/sulbactam vs. colistin as monotherapy for the treatment of multidrug resistant Acinetobacter baumannii ventilator-associated pneumonia. Journal of Infection 2008; 56:432-436

20. Levin A.S., Levy C.E., Manrique A.E., et al. Severe nosocomial infections with imipenem-resistant Acinetobacter baumannii treated with ampicillin/sulbactam. Int J Antimicrob Agents 2003; 21:58-62.

21. Cisneros J.M., Reyes M.J., Pachón J., et al. Bacteremia due to Acinetobacter baumannii: epidemiology, clinical findings, and prognostic features. Clin Infect Dis 1996; 22:1026-32.

22. Jellison T.K., Mckinnon P.S., Rybak M.J.: Epidemiology, resistance, and outcomes of Acinetobacter baumannii bacteremia treated with imipenemcilastatin or ampicillin-sulbactam. Pharmacotherapy 2001; 21:142-48.
23. Wood G.C., Hanes S.D., Croce M.A., et al. Comparison of ampicillin-sulbactam and imipenem-cilastatin for the treatment of Acinetobacter ventilator-associated pneumonia. Clin Infect Dis 2002; 34:1425-30.

24. Lodise T.P., Lomaestro B.M., Drusano G.L. Application of Antimicrobial Pharmacodynamic Concepts into Clinical Practice: Focus on b-Lactam Antibiotics. Insights from the Society of Infectious Diseases Pharmacists. Pharmacotherapy 2006; 26:1320-32.

25. Bantar C., Schell C., Posse G., et al. Comparative time-kill study of doxycycline, tigecycline, sulbactam, and imipenem against several clones of Acinetobacter baumannii. Diagn Microbiol Infect Dis. 2008; 61:309-14.

26. Lodise T.P. Jr, Lomaestro B., Drusano G.L. Piperacillin-tazobactam for Pseudomonas aeruginosa infection: clinical implications of an extended-infusion dosing strategy. Clin Infect Dis 2007; 44:357-63.

27. Jones RN, Fritsche TR, Moet GJ. In vitro potency evaluations of various piperacillin/tazobactam generic products compared with the contemporary branded (Zosyn, Wyeth) formulation. Diagn Microbiol Infect Dis 2008; 61:76-9. 